\title{
A Novel Approach to Predicting Interactions Between Microbes and Hosts
}

\author{
Jie Li ( $\boldsymbol{\sigma}_{\text {jieli@hit.edu.cn ) }}$ \\ Harbin Institute of Technology \\ Zhuo Chen \\ Harbin Institute of Technology \\ Dawei Ma \\ Harbin Institute of Technology \\ Li Zhou \\ Harbin Institute of Technology

\section{Yadong Wang} \\ Harbin Institute of Technology
}

\section{Research Article}

Keywords: human health, KATZHMDA, WBSMDA , NGRHMDA, host interactions, living organisms

Posted Date: April 13th, 2021

DOI: https://doi.org/10.21203/rs.3.rs-400365/v1

License: (c) (i) This work is licensed under a Creative Commons Attribution 4.0 International License. Read Full License 


\title{
A novel approach to predicting interactions between microbes
}

\author{
and hosts \\ Jie Li ${ }^{1, *}$, Zhuo Chen ${ }^{1}$, Dawei Ma ${ }^{1}$, Li Zhou ${ }^{1}$ and Yadong Wang ${ }^{1, *}$ \\ ${ }^{1}$ School of Computer Science and Technology, Harbin Institute of Technology, China \\ *Correspondence: jieli@hit.edu.cn, ydwang@hit.edu.cn,
}

\section{Abstract}

Increasing evidence shows that microbes are important for the protection of human health and the health of other living organisms. At the same time, microbes can cause other organisms to become sick or even die. Through microbe-host interaction, we can understand intuitively the process and mechanism of host infection by microbes. Several methods are developed to predict microbe-host interactions. However, current methods are limited by the cost of interaction verification experiments and accuracy. Therefore, there is still a need for a rapid and accurate method to predict microbe-host interaction. Here, we proposed a novel method based on Integrated Similarity, KATZ measure, and Within and Between Scores (ISKATZWBS) to predict microbe-host interactions. Experimental results show that the proposed method performs well and the AUCs are $0.946,0.981,0.954$ on the PHI-base, HPIDB, and HMDAD datasets repectively. Compared with other four state-of the-art methods: KATZHMDA, WBSMDA, NGRHMDA and NCPHMDA, the proposed method has higher prediction accuracy.

\section{Introduction}

"Microbe" is a general designation of all microorganisms that are difficult to observe by the naked eye, including bacteria, eukaryotes, archaea, and viruses [1]. On the one hand, the healthy survival of human and other living organisms is inseparable from the role of microbes. Microbes benefit the host with improved metabolic capabilities, protection from pathogens, enhancement of the immune system, and the modulation of gastrointestinal development [2]. Recent work has demonstrated that microbe composition is predictive of the efficacy of cancer immunotherapy [3]. Focusing on microbe-immunity interactions may provide insight into the principles of immune system function while facilitating precision therapeutics for systemic disease [4]. On the other hand, microbes may cause disease in the host or even death. With the development of globalization, global health care is facing an increasing number of severe challenges. For example, on April 21, 2009, the 2009 influenza $A(H 1 N 1)$, caused by influenza virus A, spread rapidly worldwide, and on June 11,2009 , the World Health Organization raised the pandemic alert level to the highest level of 6.2 [5]. As of March 12, 2020, coronavirus disease 2019 (COVID-19) has been confirmed in 125,048 
people worldwide, carrying a mortality rate of approximately $3.7 \%$, compared to a mortality rate of less than $1 \%$ from influenza [6].

By exploring the interaction between microbes and hosts, we can find effective ways to block transmission and to treat related diseases. With the rapid development of whole genome sequencing, scientists have accumulated a large amount of genetic data. There is a new understanding of biology and biological activities at the molecular level. Scientists use this accumulated data to verify a large number of microbe-host interactions and have stored these interactions in public databases such as PHI-base [7], HPIDB [8], PATRIC [9], PHISTO [10], VirHostNet [11], and Mentha [12]. However, due to the wide variety of proteins and the time and economic cost of verifying microbe-host interactions by experiment, we cannot verify all microbe-host interactions by such methods. Consequently, an increasing number of calculation methods are now used to predict microbe-host interaction relationships with the aim of helping researchers to carry out targeted experiments.

Li et al. [13] used bipartite network projection and introduced an algorithm called the Bipartite Network Module-Based Project (BNMP) to predict pathogen-host association. The method is based on bipartite network modules and integrates the module information of pathogens and hosts respectively into a bipartite network projection model to improve prediction performance. Chen et al. [14] developed a novel computational model of KATZ measure for Human Microbe-Disease Association prediction (KATZHMDA), based on the assumption that functionally similar microbes tend to have similar interaction and non-interaction patterns with non-infectious diseases and vice versa. He et al. [15] developed a novel predictive model of Graph Regularized Non-Negative Matrix Factorization for Human Microbe-Disease Association prediction (GRNMFHMDA). Chen et al [16] developed the model of Within and Between Scores for MiRNA-Disease Association (WBSMDA prediction) to predict potential miRNAs associated with various complex diseases. WBSMDA can be used to predict unknown miRNA-disease associations.

In this paper, we develop a novel approach to predict associations of microbes and hosts based on Integrated Similarity, KATZ measure, and Within and Between Scores (ISKATZWBS), which is an improvement on KATZHMDA [14] and WBSMDA [16]. In a similar way to KATZHMDA, ISKATZWBS uses Gaussian interaction profile kernel similarity and cosine similarity to obtain the similarity matrix between microorganisms and hosts. The balance factor is then added to the KATZ model, and the results of WBSMDA are used to replace the adjacency matrix of the microbe-host interaction in the KATZ model.

\section{Materials and Method}

\section{Known Bacteria-Host Associations}

The pathogen-host interactions database PHI-base (http://www.phi-base.org/index.jsp) collects data on bacteria-host interaction by manually reading the literature [7]. Users can download data from PHI-base freely and our known bacteria-host association data is downloaded from PHI-base. There are 267 bacteria, 203 hosts, and 13,802 bacteria-host protein interaction data in the dataset. After data preprocessing, we obtained 819 distinct 
confirmed interactions between bacteria and hosts. We constructed the adjacency matrix $A \in R^{n_{b} \times n_{h}}$ as the original input data for the experiment, where $n_{b}$ is the number of bacteria and $n_{h}$ the number of hosts. $A(i, j)=1$ indicates that there is a confirmed interaction between the $i$-th bacteria and the $j$-th host; otherwise $A(i, j)=0$.

\section{Gaussian Interaction Profile Kernel Similarity for Bacteria}

Based on the assumption that similar hosts may be infected by the same microbe, we calculate the similarity between bacteria and hosts separately by using Gaussian interaction profile kernel similarity. For the adjacency matrix $A$, the bacteria-host interaction is expressed as a binary: 1 means there is an interaction between the bacteria and host; otherwise $A=0$. For a given bacteria $b_{i}$, its interaction profile $I P\left(b_{i}\right)$ can be determined by the $i$-th row of the adjacency matrix $A$. The Gaussian interaction profile kernel similarity between $b_{i}$ and $b_{j}$ can be calculated by the following formulae:

$$
\begin{aligned}
K B\left(b_{i}, b_{j}\right) & =\exp \left(-\gamma_{b}\left\|I P\left(b_{i}\right)-I P\left(b_{j}\right)\right\|^{2}\right) \\
\gamma_{b} & =\gamma_{b}^{\prime} / \frac{\sum_{k=1}^{n_{b}}\left\|I P\left(b_{k}\right)\right\|^{2}}{n_{b}}
\end{aligned}
$$

In Formula (1), $n_{b}$ represents the number of bacteria in the dataset and $\gamma_{b}$ represents normalized kernel bandwidth based on $\gamma_{b}^{\prime}$ in Formula (2). Following van Laarhoven et al. [17] and Chen et al. $[14,16]$, we set $\gamma_{b}^{\prime}=1$. From the two formulae above, we know that $K B\left(b_{i}, b_{i}\right)=1$ and $0<K B\left(b_{i}, b_{j}\right)<1$. According to Xie et al. [18] and Vanunu et al. [19], when $0<K B\left(b_{i}, b_{j}\right) \leq 0.3, b_{i}$ and $b_{j}$ cannot be considered similar. When $0.6 \leq$ $K B\left(b_{i}, b_{j}\right)<1, b_{i}$ and $b_{j}$ may show significant similarity. Therefore, we use logistic function transformation from $K B\left(b_{i}, b_{j}\right)$ to $K B^{*}\left(b_{i}, b_{j}\right)$ as shown in Formula (3) to make the confidence interval of $K B\left(b_{i}, b_{j}\right)$ more reasonable.

$$
K B^{*}\left(b_{i}, b_{j}\right)=1 /\left(1+e^{c \times K B\left(b_{i}, b_{j}\right)+d}\right)
$$

Following Xie et al. [18] and Vanunu et al. [19], we set $c=-15$ and $d=\log (9999)$ so that $K B^{*}\left(b_{i}, b_{j}\right)=0.0001$ when $K B\left(b_{i}, b_{j}\right)=0$.

\section{Gaussian Interaction Profile Kernel Similarity for Host}

In a similar way to the Gaussian interaction profile kernel similarity of bacteria, for a given host $h_{i}$, its interaction profile $I P\left(h_{i}\right)$ can be determined by the $i$-th column of the adjacency matrix $A$. The Gaussian interaction profile kernel similarity between $h_{i}$ and $h_{j}$ can be calculated by the following formulae:

$$
\begin{gathered}
K H\left(h_{i}, h_{j}\right)=\exp \left(-\gamma_{b}\left\|I P\left(h_{i}\right)-I P\left(h_{j}\right)\right\|^{2}\right) \\
\gamma_{b}=\gamma_{b}^{\prime} / \frac{\sum_{k=1}^{n_{h}}\left\|I P\left(h_{k}\right)\right\|^{2}}{n_{h}} \\
K H^{*}\left(h_{i}, h_{j}\right)=1 /\left(1+e^{c * K H\left(h_{i}, h_{j}\right)+d}\right)
\end{gathered}
$$


In the above, $n_{h}$ is the number of hosts in the dataset. We set $\gamma_{b}^{\prime}=1, c=-15$, and $d=\log (9999)$, as before.

\section{Cosine Similarity for Bacteria and Host}

The Calculation of cosine similarity (i.e., the calculation of cosine similarity of disease[20], bacteria and host) is based on the assumption that if bacteria $b_{i}$ and bacteria $b_{j}$ are similar to each other, then, in the bacteria-host association matrix, pattern $A(:, i)$ (i.e., the $i$-th column of the adjacency matrix $A$ ) and pattern $A(:, j)$ (i.e., the $j$-th column of adjacency matrix $A$ ) should be similar to each other. The same assumption should also be true for host. Therefore, the cosine similarity between bacteria $b_{i}$ and bacteria $b_{j}$ can be defined as follows:

$$
\mathrm{CB}\left(b_{i}, b_{j}\right)=\frac{\mathrm{A}(:, i) * \mathrm{~A}(:, j)}{\|\mathrm{A}(:, i)\| *\|\mathrm{~A}(:, j)\|}
$$

After calculating the cosine similarity between each pair of bacteria-bacteria, the cosine similarity matrix for bacteria $C B\left(n_{b}{ }^{*} n_{b}\right)$ can be constructed. Similarly, the cosine similarity between host $h_{i}$ and host $h_{j}$ is given by:

$$
\mathrm{CH}\left(h_{i}, h_{j}\right)=\frac{\mathrm{A}(i) * \mathrm{~A}(j)}{\|\mathrm{A}(i)\| *\|\mathrm{~A}(j)\|}
$$

\section{Integrated Similarity for Bacteria and Host}

To make full use of the Gaussian interaction profile kernel similarity matrix for bacteria $K B$ and the cosine similarity matrix for bacteria $C B$, a comprehensive similarity matrix for bacteria $B S\left(n_{b} * n_{b}\right)$ was constructed by integrating the $K B$ and $C B$ similarity matrices as follows:

$$
B S=\frac{K B+C B}{2}
$$

In Formula (9), $B S\left(b_{i}, b_{j}\right)$ represents the integrated similarity between bacteria $b_{i}$ and bacteria $b$.

In the same way, the Gaussian interaction profile kernel similarity matrix for host $\mathrm{KH}$ and the cosine similarity matrix for host $\mathrm{CH}$ are integrated into a comprehensive similarity matrix for host $H S\left(n_{h} * n_{h}\right)$ as follows:

$$
H S=\frac{K H+C H}{2}
$$

In Formula (10), $H S\left(h_{i}, h_{j}\right)$ represents the integrated similarity between host $h_{i}$ and host $h_{j}$. As a result of the above, we obtain a comprehensive host similarity matrix $H S$ and a comprehensive bacteria similarity matrix $B S$, respectively. 


\section{WBSMDA}

WBSMDA(Within and Between Scores for MiRNA-Disease Association) is an approach to predict Mirna-Disease association by calculating within and between scores [16, 21]. Here we employ it to predict the bacteria-host association. According to WBSMDA, the within-score and between-score of a bacteria-host pair $\left(b_{i}, h_{j}\right)$ can be defined by the following formulae:

$$
\begin{aligned}
& S_{b}^{w}\left(b_{i}, h_{j}\right)=\max _{b_{k} \in b_{h_{j}}} B S\left(b_{i}, b_{k}\right) \\
& S_{h}^{w}\left(b_{i}, h_{j}\right)=\max _{h_{k} \in h_{b_{i}}} H S\left(h_{j}, h_{k}\right) \\
& S_{b}^{b}\left(b_{i}, h_{j}\right)=\max _{b_{k} \in b_{\bar{h}_{j}}} B S\left(b_{i}, b_{k}\right) \\
& S_{h}^{b}\left(b_{i}, h_{j}\right)=\max _{h_{k} \in h_{\bar{b}_{l}}} H S\left(h_{j}, h_{k}\right)
\end{aligned}
$$

In the above, $b_{h_{j}}$ is the group of bacteria that has interaction with $h_{j}$ in the dataset, $h_{b_{i}}$ is the group of hosts that has interaction with $b_{i}$ in the dataset, $b_{\overline{h_{j}}}$ is the group of bacteria that does not have interaction with $h_{j}$ in the dataset, and $h_{\bar{b}_{l}}$ is the group of hosts that does not have interaction with $b_{i}$ in the dataset. In short, for a given bacteria $b_{i}$, within-score is looking for the highest integrated similarity score in the group of bacteria with known interaction with a given host $h_{j}$, whereas between-score is looking for the highest integrated similarity score in the group of bacteria with no known interaction with a given host $h_{j}$. Similarly, within-score and between-score have the same meanings for a given host $h_{j}$.

We then calculate the predicted score $P\left(b_{i}, h_{j}\right)$ of the interaction between $b_{i}$ and $h_{j}$ according to the following formula:

$$
P\left(b_{i}, h_{j}\right)=\frac{S_{b}^{w}\left(b_{i}, h_{j}\right) \times S_{h}^{w}\left(b_{i}, h_{j}\right)}{S_{b}^{b}\left(b_{i}, h_{j}\right) \times S_{h}^{b}\left(b_{i}, h_{j}\right)}
$$

In addition, for a given bacteria $b$ that has no known interaction with any host in the dataset, the predicted score $P\left(b, h_{j}\right)$ can be calculated by the following formula:

$$
P\left(b, h_{j}\right)=\frac{S_{b}^{w}\left(b, h_{j}\right)}{S_{b}^{b}\left(b, h_{j}\right)}
$$

Similarly, for a given host $h$ that has no known interaction with any bacteria in the dataset, the predicted score $P\left(b_{i}, h\right)$ can be calculated by the following formula:

$$
P\left(b_{i}, h\right)=\frac{S_{h}^{w}\left(b_{i}, h\right)}{S_{h}^{b}\left(b_{i}, h\right)}
$$

Finally, we obtain the prediction score matrix $P \in R^{n_{b} \times n_{h}}$ by WBSMDA, where $P(i, j)$ is the prediction score for a given bacteria $b_{i}$ and a given host $h_{j}$.

\section{ISKATZWBS}

KATZ measure is used for social network prediction [22], disease-gene association prediction [23], IncRNA-disease association prediction [24, 25], and microbe-disease prediction [14, 18] KATZ is a link prediction method that calculates the similarity of nodes in a heterogeneous 
network through random walk. The number of walks between nodes and walk lengths in the network is effective similarity metrics of KATZ Measure. Here, we proposed a new approach IKATZWBS by improving KATZ measure to predict bacteria-host association. The overall process of ISKATZWBS is shown in Figure 1.

First, we calculate the bacteria gaussian interaction profile kernel similarity matrix $\mathrm{KB}$, the bacteria cosine similarity matrix $\mathrm{CB}$, the host gaussian interaction profile kernel similarity matrix $\mathrm{KH}$ and the host cosine similarity matrix $\mathrm{CH}$ respectively, based on adjacency matrix $\mathrm{A}$. Then, in order to make full use of bacteria and host similarity information, two comprehensive similarity matrixes for bacteria and Hosts BS and HS are constructed respectively. Next, due to the origin adjacency matrix $A$ was too sparse, a bacteria-host association prediction score matrix $P$ is calculated using WBSMDA which contains more bacteria-host associations. After that, we form a heterogeneous network $A^{*}$ by integrating WBSMDA prediction score matrix $\mathrm{P}$, bacteria integrated similarity matrix BS and host integrated similarity matrix HS. In addition, in order to balance the contribution of the integrated similarity for host and the integrated similarity for bacteria during the random walk, we introduced the balance factor $a$ and $b(a, b \in[0,1])$ to control the contribution of host integrated similarity and bacteria integrated similarity during the process of random walk. Thus, the heterogeneous network $A^{*}$ can be defined as follow:

$$
A^{*}=\left[\begin{array}{cc}
a \times B S & P \\
P^{T} & b \times H S
\end{array}\right]
$$

The whole random walking process can be expressed by the formula (19):

$$
S=\sum_{l \geq 1} \delta^{l} A^{* l}=\left(E-\delta A^{*}\right)^{-1}-E
$$

In Formula (19), $A^{* l}$ is the $l$-th power of $A^{*}$, the element $A^{* l}\left(b_{i}, h_{j}\right)$ which is the $i$-th row and $j$-th column of $A^{*}$ means the number of $l$-length walks between bacteria $b_{i}$ and host $h_{j} . \delta \in(0,1)$ is the dampen factor of walks of different length, which means $\delta^{l}$ is the dampen factor of $l$-length walks. $S \in R^{\left(n_{b}+n_{h}\right) \times\left(n_{b}+n_{h}\right)}$ is the final score matrix obtained by the heterogeneous network matrix $A^{*}$ after $l$-length walks, and $E$ is the identity matrix. $S$ can be divided into four sub-matrices according to Formula (20):

$$
S=\left[\begin{array}{ll}
S_{1} & S_{2} \\
S_{3} & S_{4}
\end{array}\right]
$$

Obviously, $S_{1} \in R^{n_{b} \times n_{b}}, S_{2} \in R^{n_{b} \times n_{h}}, S_{3} \in R^{n_{h} \times n_{b}}$, and $S_{4} \in R^{n_{h} \times n_{h}}$. Obviously, $S_{2}$ is the final prediction score matrix which we want and the element $S_{2}(i, j)$ of $S_{2}$ is the prediction score of bacteria $b_{i}$ and host $h_{j}$. According to Chen et. [14], when the length of walk $l=2$ the KATZ Measure performance is the best. Therefor we also set $l=2$ and the whole random walk process can be simplified as follow:

$$
S_{l=2}=\delta \times P+\delta^{2}(a \times B S \times P+P \times H S \times b)
$$

In Formula (21) $S_{l=2} \in R^{n_{b} \times n_{h}}$ is the final bacteria - host association prediction result matrix, the element $S_{l=2}\left(b_{i}, h_{j}\right)$ is the $i$-th row and $j$-th column of $S_{l=2}$ which means the final association prediction score between bacteria $b_{i}$ and host $h_{j}$. 


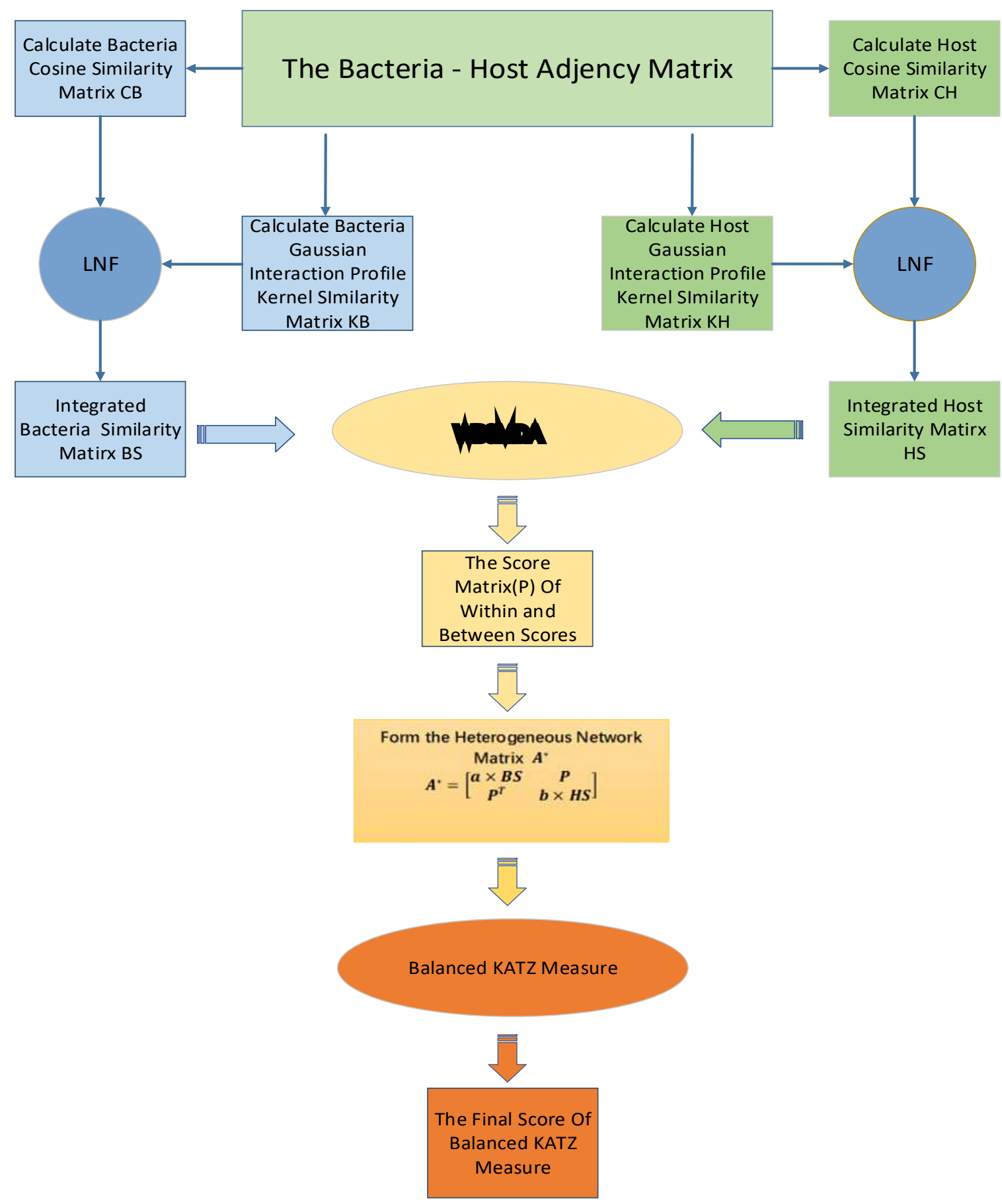

Figure 1. The process of ISKATZWBS 


\section{Results}

\section{Performance Evaluation}

We downloaded bacterial-host interaction data from PHI-base (http://www.phi-base.org/ downloadLink.htm) as the verification dataset and used Leave One Out Cross Validation (LOOCV) [26] to test the prediction performance of ISKATZWBS on the verification dataset. In LOOCV, each bacteria-host interaction is used in turn as a test dataset and other bacteriahost interactions are used as a training dataset. In each round of LOOCV, we used ISKATZWBS to make predictions on the training dataset. After LOOCV, we plotted Receiver Operating Characteristic (ROC) curves and Precision Recall (PR) curves to evaluate the performance of the algorithm. In order to display the performance evaluation results more intuitively, we calculated the AUC (the area enclosed by the ROC curve and the coordinate axis) and AUPR (the area enclosed by the PR curve and the coordinate axis), respectively.

With our method, the balance factors $a$ and $b$ are important parameters that affect the final result. We take values $a$ and $b$ from 0 to 1 in steps of 0.1 respectively. We then use data from PHI-base for LOOCV to select the parameters with the best performance results. As shown in Figure 2 and Figure 3, when $a=0.3$ and $b=0.1$, the ISKATZWBS prediction has the best performance when AUC $=0.947$ and $A U P R=0.429$.

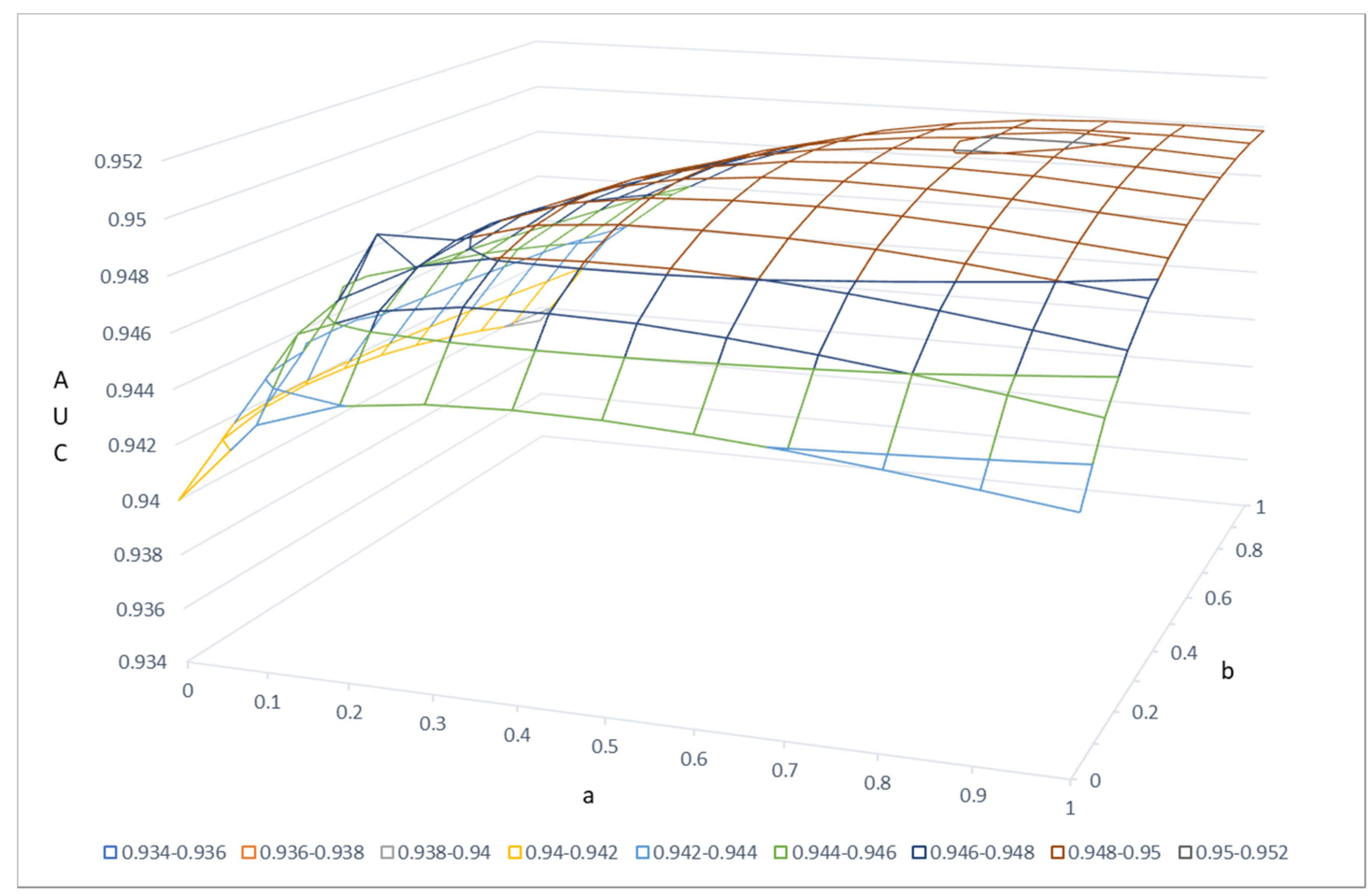

Figure 2. The LOOCV AUC results of ISKATZWBS with different parameters $a$ and $b$ 


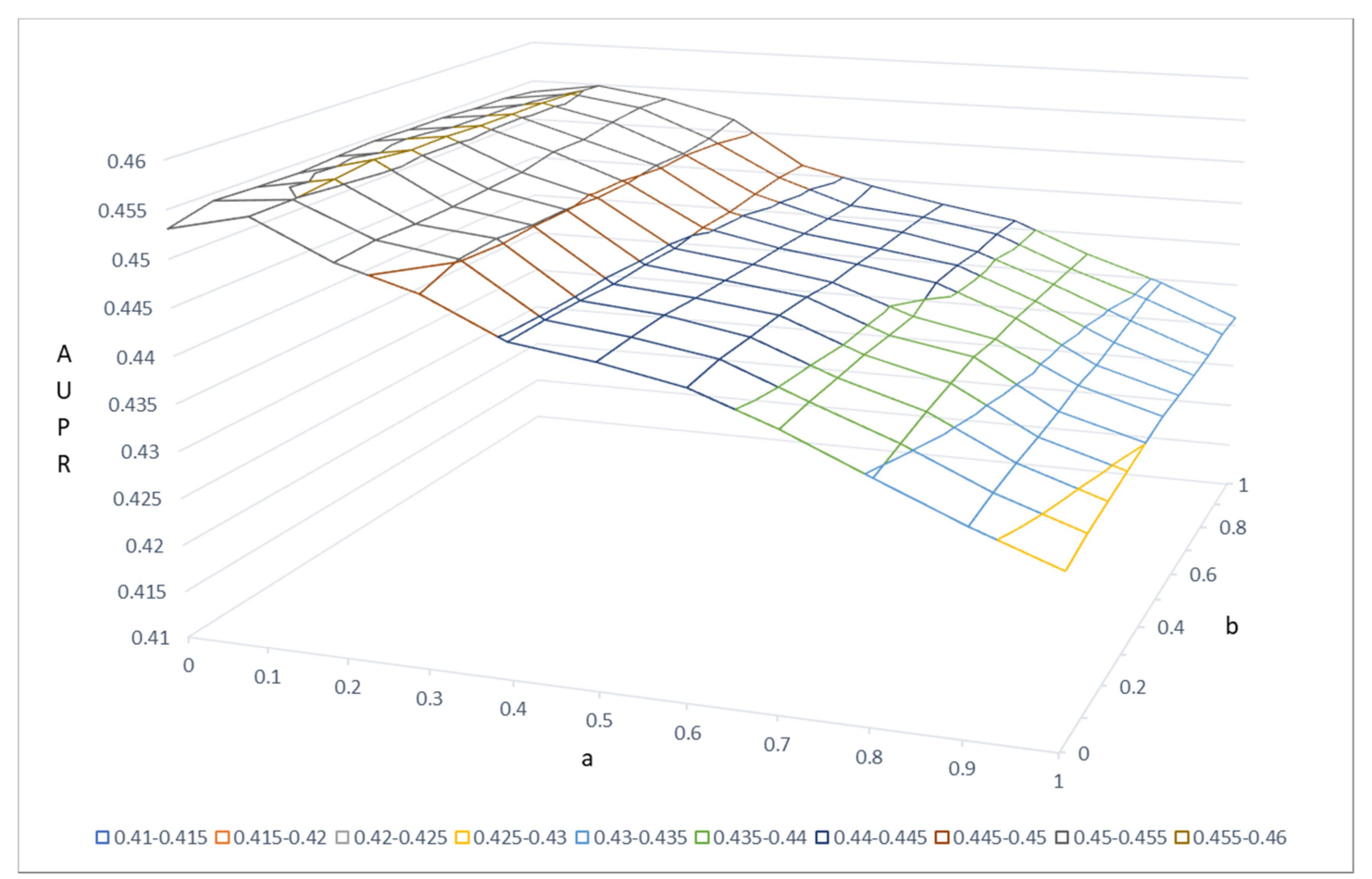

Figure 3. The LOOCV AUPR results of ISKATZWBS with different parameters $a$ and $b$

\section{Comparison with Existing Methods}

We compared ISKATZWBS with four state-of the-art methods - KATZHMDA [14], WBSMDA [16], NGRHMDA[27] and NCPHMDA[29] - by using LOOCV with data from PHI-base [7], HPIDB [8], HMDAD [28]. The data statistics of these databases are shown in Table 1.

Table 1. Data statistics of PHI-base, HPIDB, and HMDAD

\begin{tabular}{l|l|l}
\hline Databases & Database content & $\begin{array}{l}\text { Data volume (after data } \\
\text { preprocessing) }\end{array}$ \\
\hline PHI-base & Bacteria-host interaction & $\begin{array}{l}270 \text { bacteria, 212 hosts, and } 855 \\
\text { distinct bacteria-host interactions. }\end{array}$ \\
\hline HPIDB & Virus-host interaction & $\begin{array}{l}668 \text { viruses, } 66 \text { hosts, and 967 distinct } \\
\text { virus-host interactions. }\end{array}$ \\
\hline HMDAD & Human microbe-disease association & $\begin{array}{l}292 \text { microbes, } 39 \text { diseases, and } 450 \\
\text { distinct microbe-disease associations. }\end{array}$ \\
\hline
\end{tabular}

As above, we used LOOCV to determine the performance of these four methods. The LOOCV AUC results for ISKATZWBS, KATZHMDA, WBSMDA, NGRHMDA and NCPHMDA with data from PHI-base were $0.946,0.790,0.833,0.831$ and 0.879 respectively. The LOOCV AUC results for ISKATZWBS, KATZHMDA, WBSMDA, NGRHMDA and NCPHMDA with data from HPIDB were $0.981,0.943,0.940,0.922$ and 0.937 respectively. The LOOCV AUC results for ISKATZWBS, KATZHMDA, WBSMDA, NGRHMDA and NCPHMDA with data from HMDAD were $0.954,0.880,0.931,0.862$ and 0.929 respectively. The LOOCV AUC results are shown in Figure 4. 


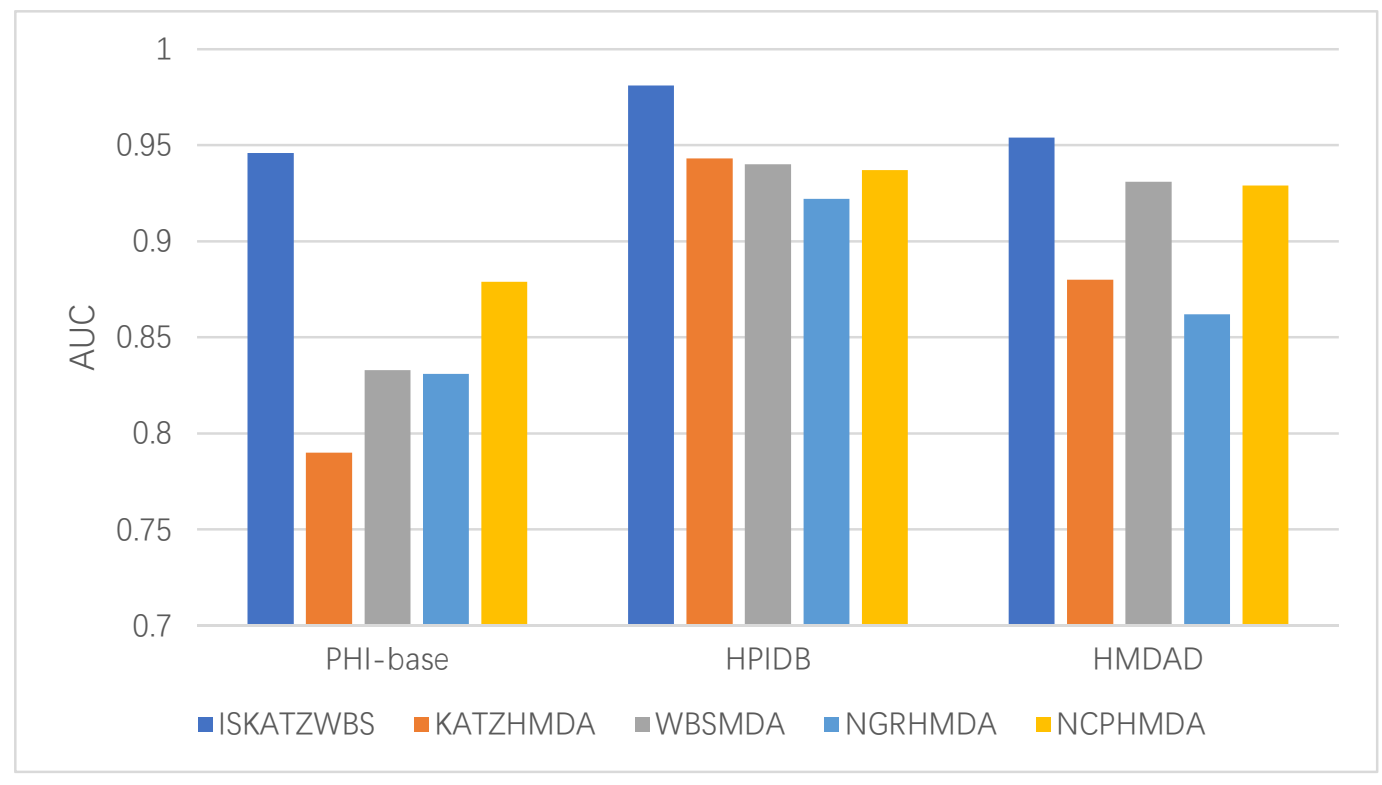

Figure 4. The AUC results of five methods on three data

The LOOCV AUPR results for ISKATZWBS, KATZHMDA, WBSMDA, NGRHMDA and NCPHMDA with data from PHI-base were $0.456,0.142,0.271,0.108$ and 0.292 respectively. The LOOCV AUPR results for ISKATZWBS, KATZHMDA, WBSMDA, NGRHMDA and NCPHMDA with data from HPIDB were $0.754,0.679,0.635,0.254$ and 0.296 respectively. The LOOCV AUPR results for ISKATZWBS, KATZHMDA, WBSMDA, NGRHMDA and NCPHMDA with data from HMDAD were $0.630,0.487,0.483,0.481$ and 0.575 respectively. The LOOCV AUPR results are shown in Figure 5.

Figures 4 and 5 show that ISKATZWBS performs better than other four methods.

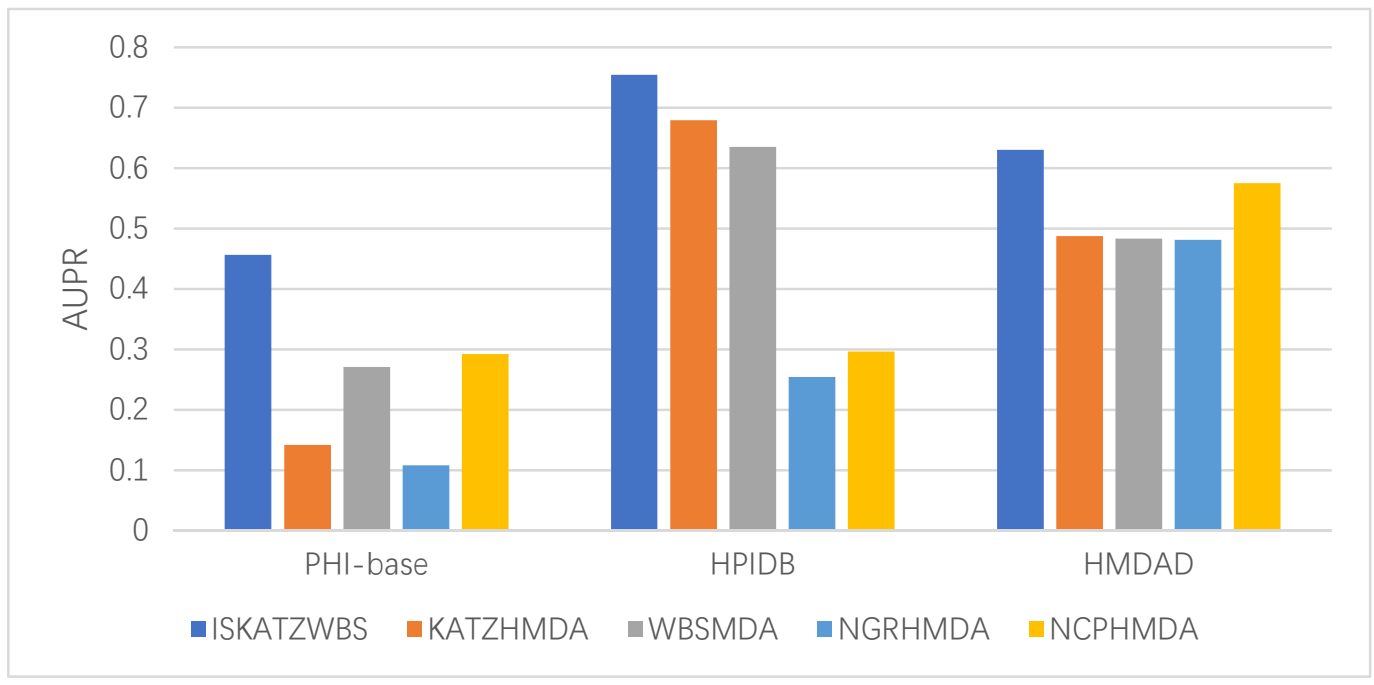

Figure 5. The AUPR results of five methods on three data 


\section{Validation via Biological Evidence}

Due to data mining technology and cost constraints, PHI-base cannot cover all bacterialhost interaction data. In order to further verify the prediction performance of ISKATZWBS, we selected the top 20 potential bacteria-host relationship pairs and searched the literature to find corresponding evidences to prove their potential interaction relationship. We found that 17 of the 20 potential bacteria-host interactions provided biological evidence, as summarized in Table 2 (note that the potential prediction scores ranked at 8, 9, and 13 lacked confirmation in the literature). The details are as follows, with the evidence listed in order of prediction ranking:

1) Trichophyton mentagrophytes-Mus musculus. Mackenzie [30] reported an incident in which children were infected with Trichophyton mentagrophytes by mice (Mus musculus). In the subsequent experiment, Trichophyton mentagrophytes were isolated from 104 of 147 mice (Mus musculus).

2) Vibrio harveyi-Homo sapiens. Vibrio harveyi is an opportunistic human pathogen that may cause gastroenteritis, severe necrotizing soft-tissue infections, and primary septicemia, with a potentially high rate of lethality. Kang et al. [31] isolated Vibrio harveyi from seawater to study their drug resistance.

3) Edwardsiella tarda-Homo sapiens. Jordan et al. [32] found that humans will have symptoms similar to those caused by Salmonellae after being infected with Edwardsiella tarda.

4) Staphylococcus aureus-Drosophila melanogaster. Needham et al. [33] found that Drosophila melanogaster can be used to verify the virulence of Staphylococcus aureus.

5) Bipolaris victoriae-Triticum. Momtaz et al. [34] infected Triticum through Bipolaris victoriae to discover the relationship between Triticum infected by bipolaris leaf blight.

6) Venturia inaequalis-Mus musculus. Following previous reports that the G143A mutation of cytochrome b caused resistance of Venturia inaequalis to a strobilurin-related inhibitor in mice (Mus musculus) mitochondria, Zheng et al. [35] studied the characterization of laboratory mutants of Venturia inaequalis resistant to the strobilurin-related fungicide kresoxim-methyl.

7) Colletotrichum lagenarium-Nicotiana benthamiana. Takano et al. [36] found that the Colletotrichum lagenarium wild strain 104-T is able to infect Nicotiana benthamiana, which is not closely related to cucumber.

10) Verticillium fungicola-Arabidopsis thaliana. Athey-Pollard et al. [37] compared the percentage homology of Verticillium fungicola and Agaricus bisporus elF4E amino acid sequences with those isolated from Arabidopsis thaliana and other hosts.

11) Alternaria citri-Citrus reticulata. Kono et al. [38] studied host-selective toxins produced by a pathotype of Alternaria citri, a fungus that produces brown spot disease of Dancy tangerine (Citrus reticulata) and other mandarin cultivars.

12) Escherichia coli-Danio rerio. Stones et al. [39] described the establishment of a vertebrate model for foodborne EHEC infection, using larval zebrafish (Danio rerio) as a host and the protozoan prey Paramecium caudatum as a vehicle.

14) Fusarium sporotrichioides-Triticum aestivum. Asano et al. [40] noted that certain 
graminaceous plants such as Zea mays and Triticum aestivum serve as hosts for Fusarium sporotrichioides, and went on to study the molecular interactions between the host plants and F. sporotrichioides.

15) Beauveria bassiana-Arabidopsis thaliana. Raad et al. [41] assessed the effects of two Beauveria bassiana strains (BG11 and FRh2) on the growth of Arabidopsis thaliana and its resistance against two herbivore species and a phytopathogen.

16) Sclerotinia sclerotiorum-Nicotiana tabacum. Garriz et al. [42] carried out a preliminary evaluation of the potential of polyamine biosynthesis inhibition as a strategy for the control of plant diseases initiated by Sclerotinia sclerotiorum ascospores, using tobacco (Nicotiana tabacum) leaf discs as an experimental system.

17) Cryptococcus neoformans-Gallus gallus. Kuroki et al. [43] successfully isolated Cryptococcus neoformans from chicken (Gallus gallus) feces in suburban areas of Thailand.

18) Acinetobacter nosocomialis-Homo sapiens. Visca et al. [44] discussed the infection mechanism and threats of Acinetobacter nosocomialis and other Acinetobacter species to humans.

19) Serratia marcescens-Mus musculus. Iwaya et al. [45] studied the clinical application and evaluation of rapid and quantitative detection of blood Serratia marcescens by a real-time PCR assay in a mouse (Mus musculus) infection model.

20) Alternaria alternata-Eriobotrya japonica. Tziros [46] reported that Alternaria alternata can cause leaf spot and fruit rot on loquat (Eriobotrya japonica) in Greece.

Table 2. The potential bacteria-host interaction prediction results of ISKATZWBS

\begin{tabular}{l|l|l|l}
\hline Order & Bacteria & Hosts & Evidence \\
\hline 1 & $\begin{array}{l}\text { Trichophyton } \\
\text { mentagrophytes }\end{array}$ & Mus musculus & DOl:10.1080/00362176285190351 \\
\hline 2 & Vibrio harveyi & Homo sapiens & DOI:10.1016/j.marpolbul.2014.07.008 \\
\hline 3 & Edwardsiella tarda & Homo sapiens & DOl:10.7326/0003-4819-70-2-283 \\
\hline 4 & $\begin{array}{l}\text { Staphylococcus } \\
\text { aureus }\end{array}$ & $\begin{array}{l}\text { Drosophila } \\
\text { melanogaster }\end{array}$ & DOI:10.1099/mic.0.27116-0 \\
\hline 5 & Bipolaris victoriae & Triticum & DOl:10.3329/jbas.v43i1.42228 \\
\hline 6 & Venturia inaequalis & Mus musculus & DOl:10.1007/s002940000147 \\
\hline 7 & $\begin{array}{l}\text { Colletotrichum } \\
\text { lagenarium }\end{array}$ & $\begin{array}{l}\text { Nicotiana } \\
\text { benthamiana }\end{array}$ & DOI:10.1111/j.1365-2958.2006.05080.x \\
\hline 8 & $\begin{array}{l}\text { Pyrenopeziza } \\
\text { brassicae }\end{array}$ & $\begin{array}{l}\text { Solanum } \\
\text { lycopersicum }\end{array}$ & Unconfirmed \\
\hline 9 & Kingella kingae & $\begin{array}{l}\text { Caenorhabditis } \\
\text { elegans }\end{array}$ & Unconfirmed \\
\hline 10 & Verticillium fungicola & $\begin{array}{l}\text { Arabidopsis } \\
\text { thaliana }\end{array}$ & DOl:10.1023/A:1021318524857 \\
\hline 11 & Alternaria citri & Citrus reticulata & DOl:10.1271/bbb1961.50.1597 \\
\hline 12 & Escherichia coli & Danio rerio & DOI:10.1128/mSphereDirect.00365-17 \\
\hline 13 & $\begin{array}{l}\text { Streptococcus } \\
\text { parauberis }\end{array}$ & Triticum aestivum & Unconfirmed \\
\hline 14 & Fusarium & Triticum aestivum & DOl:10.1186/1477-5956-10-61 \\
\hline
\end{tabular}




\begin{tabular}{l|l|l|l}
\hline & sporotrichioides & & \\
\hline 15 & Beauveria bassiana & $\begin{array}{l}\text { Arabidopsis } \\
\text { thaliana }\end{array}$ & DOI:10.3389/fmicb.2019.00615 \\
\hline 16 & $\begin{array}{l}\text { Sclerotinia } \\
\text { sclerotiorum }\end{array}$ & $\begin{array}{l}\text { Nicotiana } \\
\text { tabacum }\end{array}$ & DOI:10.1046/j.1469-8137.2003.00983.x \\
\hline 17 & $\begin{array}{l}\text { Cryptococcus } \\
\text { neoformans }\end{array}$ & Gallus gallus & DOI:10.1002/yea.1112 \\
\hline 18 & $\begin{array}{l}\text { Acinetobacter } \\
\text { nosocomialis }\end{array}$ & Homo sapiens & DOI:10.1002/iub.600 \\
\hline 19 & Serratia marcescens & Mus musculus & DOI:10.1016/j.femsle.2005.05.041 \\
\hline 20 & Alternaria alternata & $\begin{array}{l}\text { Eriobotrya } \\
\text { japonica }\end{array}$ & DOI:10.1007/s13314-013-0112-z \\
\hline
\end{tabular}

\section{Discussion}

In this paper, we introduced ISKATZWBS, a novel approach to predicting associations between microbes and hosts, based on integrated similarity, balanced KATZ measure, and within- and between-scores. ISKATZWBS calculates the Gaussian interaction profile kernel similarity and cosine similarity of bacteria and hosts respectively, based on the bacteria-host interaction adjacency matrix. We then integrate the Gaussian interaction profile kernel similarity and cosine similarity of bacteria and hosts, respectively. The within- and between-score matrix obtained by WBSMDA and the bacteria(host) integrated similarity are used in the balanced KATZ measure to predict potential bacteria-host interaction with high accuracy. To prove the universality of our algorithm, we compared ISKATZWBS with KATZHMDA, WBSMDA, NGRHMDA and NCPHMDA by using LOOCV with data from PHI-base, HPIDB and HMDAD. Experimental results show that ISKATZWBS has reliable prediction performance. With minor modifications, ISKATZWBS can also be used to predict other biological associations such as miRNA-disease, IncRNA-disease, drug-target, genedisease, and drug-cell line interactions.

\section{Data Availability Statement}

Publicly available datasets were analyzed in this study. This data can be downloaded from PHI-base (http://www.phi-base.org/downloadLink.htm), HPIDB (https://hpidb.igbb.msstate.edu/about.html), and HMDAD (http://www.cuilab.cn/hmdad).

\section{Declaration}

Ethics approval and consent to participate -Not applicable

\section{Consent for publication}

Availability of data and materials - Publicly available datasets were analyzed in this study. This data can be downloaded from PHI-base (http://www.phi-base.org/downloadLink.htm), HPIDB 
(https://hpidb.igbb.msstate.edu/about.html), and HMDAD (http://www.cuilab.cn/hmdad).

Author Contributions: Conceptualization, Jie Li; methodology, Jie Li, Zhuo Chen and Dawei Ma; software, Zhuo Chen and Dawei Ma; data curation, Zhuo Chen and Dawei Ma; writing - original draft preparation, Jie Li, Zhuo Chen and Dawei Ma; writing - review and editing, Jie Li, Dawei Ma.; supervision, Jie Li; project administration, Jie Li; funding acquisition, Jie Li and Yadong Wang All authors have read and agreed to the published version of the manuscript."

Funding: This research was funded by the National Key Research and Development Program of China, grant number 2016YFC0901905.

Data Availability Statement: Publicly available datasets were analyzed in this study. This data can be downloaded from PHI-base (http://www.phi-base.org/downloadLink.htm), HPIDB (https://hpidb.igbb.msstate.edu/about.html), and HMDAD (http://www.cuilab.cn/hmdad).

Acknowledgments: In this section, you can acknowledge any support given which is not covered by the author contribution or funding sections. This may include administrative and technical support, or donations in kind (e.g., materials used for experiments).

Conflicts of Interest: The authors declare no competing interests as defined by BMC, or other interests that might be perceived to influence the results and/or discussion reported in this paper.

Acknowledgements -Not applicable

\section{References}

[1] WU C, GAO R, ZHANG D, et al. PRWHMDA: human microbe-disease association prediction by random walk on the heterogeneous network with PSO []]. International Journal of Biological Sciences, 2018, 14(8): 849-57.

[2] VENTURA M, O'FLAHERTY S, et al. Genome-scale analyses of health-promoting bacteria: probiogenomics []]. Nature Reviews Microbiology, 2009, 7(1): 61-71.

[3] HELMINK B A, KHAN M A W, HERMANN A, et al. The microbiome, cancer, and cancer therapy []]. Nature Medicine, 2019, 25(3): 377-88.

[4] SPENCER S P, FRAGIADAKIS G K, SONNENBURG J L. Pursuing human-relevant gut microbiota-immune interactions []]. Immunity, 2019, 51(2): 225-39.

[5] SISTON A M, RASMUSSEN S A, HONEIN M A, et al. Pandemic 2009 influenza A (H1N1) virus illness among pregnant women in the United States []]. Jama, 2010, 303(15): 1517-25.

[6] MEHTA P, MCAULEY D F, BROWN M, et al. COVID-19: consider cytokine storm syndromes and immunosuppression []]. The Lancet, 2020, 395(10229): 1033-4.

[7] URBAN M, CUZICK A, SEAGER J, et al. PHI-base: the pathogen-host interactions database []]. Nucleic Acids Research, 2020, 48(D1): D613-D20.

[8] AMMARI M G, GRESHAM C R, MCCARTHY F M, et al. HPIDB 2.0: a curated database for host-pathogen interactions []]. Database, 2016.

[9] WATTAM A R, DAVIS J J, ASSAF R, et al. Improvements to PATRIC, the all-bacterial bioinformatics database and analysis resource center [J]. Nucleic Acids Research, 2016, 45(D1): D535-D42.

[10] DURMUŞ TEKIR S, ÇAKIR T, ARDIÇ E, et al. PHISTO: pathogen-host interaction search tool []]. Bioinformatics, 2013, 29(10): 1357-8.

[11] GUIRIMAND T, DELMOTTE S, NAVRATIL V. VirHostNet 2.0: surfing on the web of virus/host molecular interactions data []]. Nucleic Acids Research, 2014, 43(D1): D583-D7. 
[12] CALDERONE A, CASTAGNOLI L, CESARENI G. Mentha: a resource for browsing integrated protein-interaction networks []]. Nature Methods, 2013, 10(8): 690.

[13] LI J, WANG S, CHEN Z, et al. A bipartite network module-based project to predict pathogenhost association [J]. Frontiers in Genetics, 2020, 10(1357).

[14] CHEN X, HUANG Y A, YOU Z H, et al. A novel approach based on KATZ measure to predict associations of human microbiota with non-infectious diseases []]. Bioinformatics, 2016, btw715.

[15] HE B-S, PENG L-H, LI Z. Human microbe-disease association prediction with graph regularized non-negative matrix factorization []]. Frontiers in Microbiology, 2018, 9(2560).

[16] CHEN X, YAN C C, ZHANG X, et al. WBSMDA: within and between score for MiRNA-disease association prediction []]. Scientific Reports, 2016, 6(21106).

[17] TWAN V L, NABUURS S B, ELENA M. Gaussian interaction profile kernels for predicting drugtarget interaction []]. Bioinformatics, 2011, 21.

[18] XIE M, LIU X, LI S. A novel approach based on bipartite network recommendation and KATZ model to predict potential microbe-disease associations []]. Frontiers in Genetics, 2019, 10(1147).

[19] VANUNU O, MAGGER O, RUPPIN E, et al. Associating genes and protein complexes with disease via network propagation []]. PLoS Computational Biology, 2010, 6(1).

[20] XIE G, MENG T, LUO Y, LIU Z. SKF-LDA: similarity kernel fusion for predicting IncRNA-disease association. Molecular Therapy - Nucleic Acids, 2019, 18: 45-55.

[21] SHI J Y, LIU Z, HUI Y, et al. Predicting drug-target interactions via within-score and between-score [J]. BioMed Research International, 2015, 2015(1-9).

[22] KATZ L. A new status index derived from sociometric analysis [J]. Psychometrika, 1953, 18(1): 39-43.

[23] YANG X, GAO L, GUO X, et al. A network based method for analysis of IncRNA-disease associations and prediction of IncRNAs implicated in diseases []]. PloS One, 2014, 9(1): e87797.

[24] CHEN X. KATZLDA: KATZ measure for the IncRNA-disease association prediction []]. Scientific Reports, 2015, 5(1): 16840.

[25] CHEN X. Predicting IncRNA-disease associations and constructing IncRNA functional similarity network based on the information of MiRNA [J]. Scientific Reports, 2015, 5(13186).

[26] KOHAVI R. A study of cross-validation and bootstrap for accuracy estimation and model selection [C]. Proceedings of the IJCAI F, 1995. Montreal, Canada.

[27] Yu-An Huang1, Zhu-Hong You, et al.NGRHMDA: Prediction of microbe-disease association from the integration of neighbor and graph with collaborative recommendation model[]]. Translational Medicine, 2017, 15: 209.

[28] MA W, ZHANG L, ZENG P, et al. An analysis of human microbe-disease associations []]. Briefings in Bioinformatics, 2017, 18(1): 85-97.

[29] Wenzheng Bao, Zhichao Jiang and De-Shuang Huang*:Novel human microbe-disease association prediction using network consistency projection[i]. Briefings in Bioinformatics, 2017, 18(16): 173-181

[30] MACKENZIE D. Trichophyton mentagrophytes in mice: infections of humans and incidence amongst laboratory animals []]. Sabouraudia, 1962, 1(3): 178-82.

[31] KANG C-H, KIM Y, OH S J, et al. Antibiotic resistance of vibrio harveyi isolated from seawater in Korea []]. Marine Pollution Bulletin, 2014, 86(1-2): 261-5.

[32] JORDAN G W, HADLEY W K. Human infection with edwardsieila tarda []]. Annals of Internal Medicine, 1969, 70(2): 283-8. 
[33] NEEDHAM A J, KIBART M, CROSSLEY H, et al. Drosophila melanogaster as a model host for Staphylococcus aureus infection []]. Microbiology, 2004, 150(7): 2347-55.

[34] MOMTAZ M S, SHAMSI S, DEY T K. Association of bipolaris and drechslera species with bipolaris leaf blight (BPLB) infected wheat leaves [J]. Journal of Bangladesh Academy of Sciences, 2019, 43(1): 11-6.

[35] ZHENG D, OLAYA G, KÖLLER W. Characterization of laboratory mutants of venturia inaequalis resistant to the strobilurin-related fungicide kresoxim-methyl [J]. Current Genetics, 2000, 38(3): 148-55.

[36] TAKANO Y, TAKAYANAGI N, HORI H, et al. A gene involved in modifying transfer RNA is required for fungal pathogenicity and stress tolerance of colletotrichum lagenarium [J]. Molecular Microbiology, 2006, 60(1): 81-92.

[37] ATHEY-POLLARD A, KIRBY M, POTTER S, et al. Comparison of partial sequence of the cap binding protein (elF4E) isolated from agaricus bisporus and its pathogen verticillium fungicola []]. Mycopathologia, 2003, 156(1): 19-23.

[38] KONO Y, GARDNER J, SUZUKI Y, et al. Studies on host-selective toxins produced by a pathotype of alternaria citri causing brown spot disease of mandarins []]. Agricultural and Biological Chemistry, 1986, 50(6): 1597-606.

[39] STONES D H, FEHR A G, THOMPSON L, et al. Zebrafish (danio rerio) as a vertebrate model host to study colonization, pathogenesis, and transmission of foodborne escherichia coli O157 []]. mSphere, 2017, 2(5): e00365-17.

[40] ASANO T, KIMURA M, NISHIUCHI T. The defense response in arabidopsis thaliana against fusarium sporotrichioides [J]. Proteome Science, 2012, 10(1): 61.

[41] RAAD M, GLARE T R, BROCHERO H L, et al. Transcriptional reprogramming of arabidopsis thaliana defence pathways by the entomopathogen beauveria bassiana correlates with resistance against a fungal pathogen but not against insects []]. Frontiers in Microbiology, 2019, 10(615).

[42] GÁRRIZ A, DALMASSO M C, MARINA M, et al. Polyamine metabolism during the germination of sclerotinia sclerotiorum ascospores and its relation with host infection []]. New Phytologist, 2004, 161(3): 847-54.

[43] KUROKI M, PHICHAICHUMPON C, YASUOKA A, et al. Environmental isolation of cryptococcus neoformans from an endemic region of HIV-associated cryptococcosis in Thailand [J]. Yeast, 2004, 21(10): 809-12.

[44] VISCA P, SEIFERT H, TOWNER K J. Acinetobacter infection-an emerging threat to human health []]. IUBMB Life, 2011, 63(12): 1048-54.

[45] IWAYA A, NAKAGAWA S, IWAKURA N, et al. Rapid and quantitative detection of blood serratia marcescens by a real-time PCR assay: its clinical application and evaluation in a mouse infection model []]. FEMS Microbiology Letters, 2005, 248(2): 163-70.

[46] TZIROS G T. Alternaria alternata causes leaf spot and fruit rot on loquat (eriobotrya japonica) in Greece []]. Australasian Plant Disease Notes, 2013, 8(1): 123-4. 


\section{Figures}

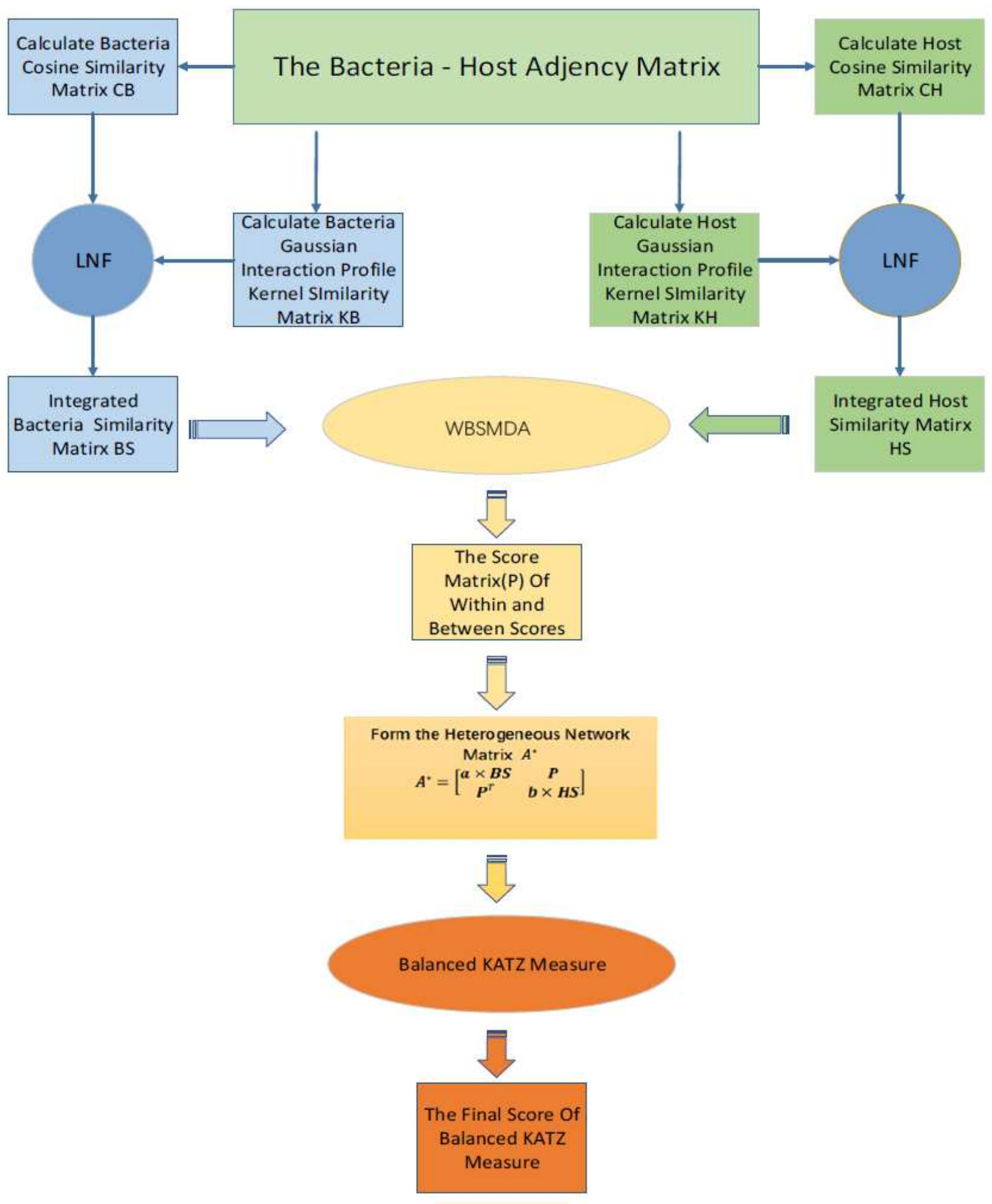

\section{Figure 1}

The process of ISKATZWBS 


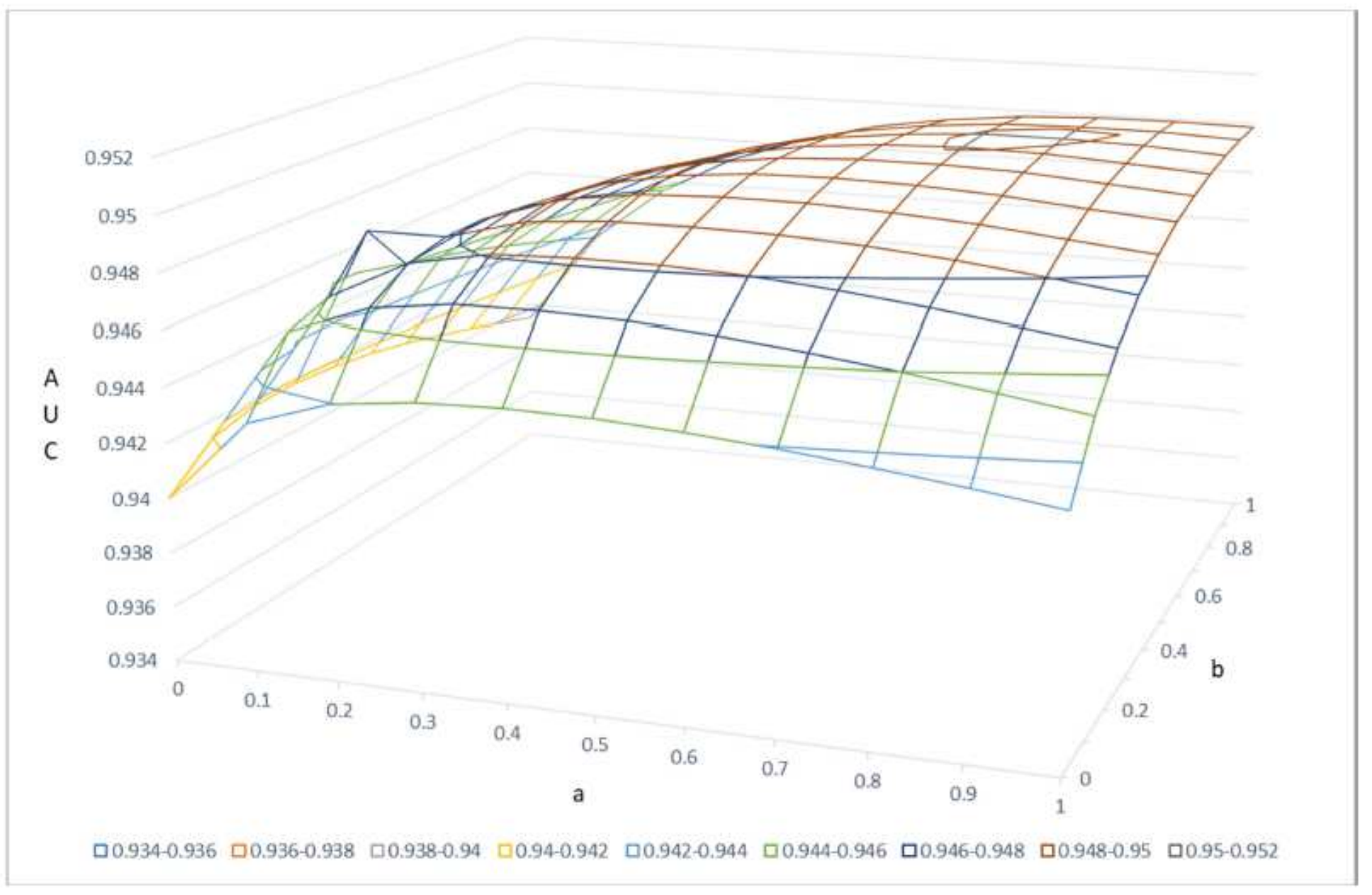

\section{Figure 2}

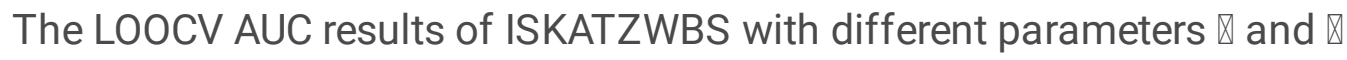




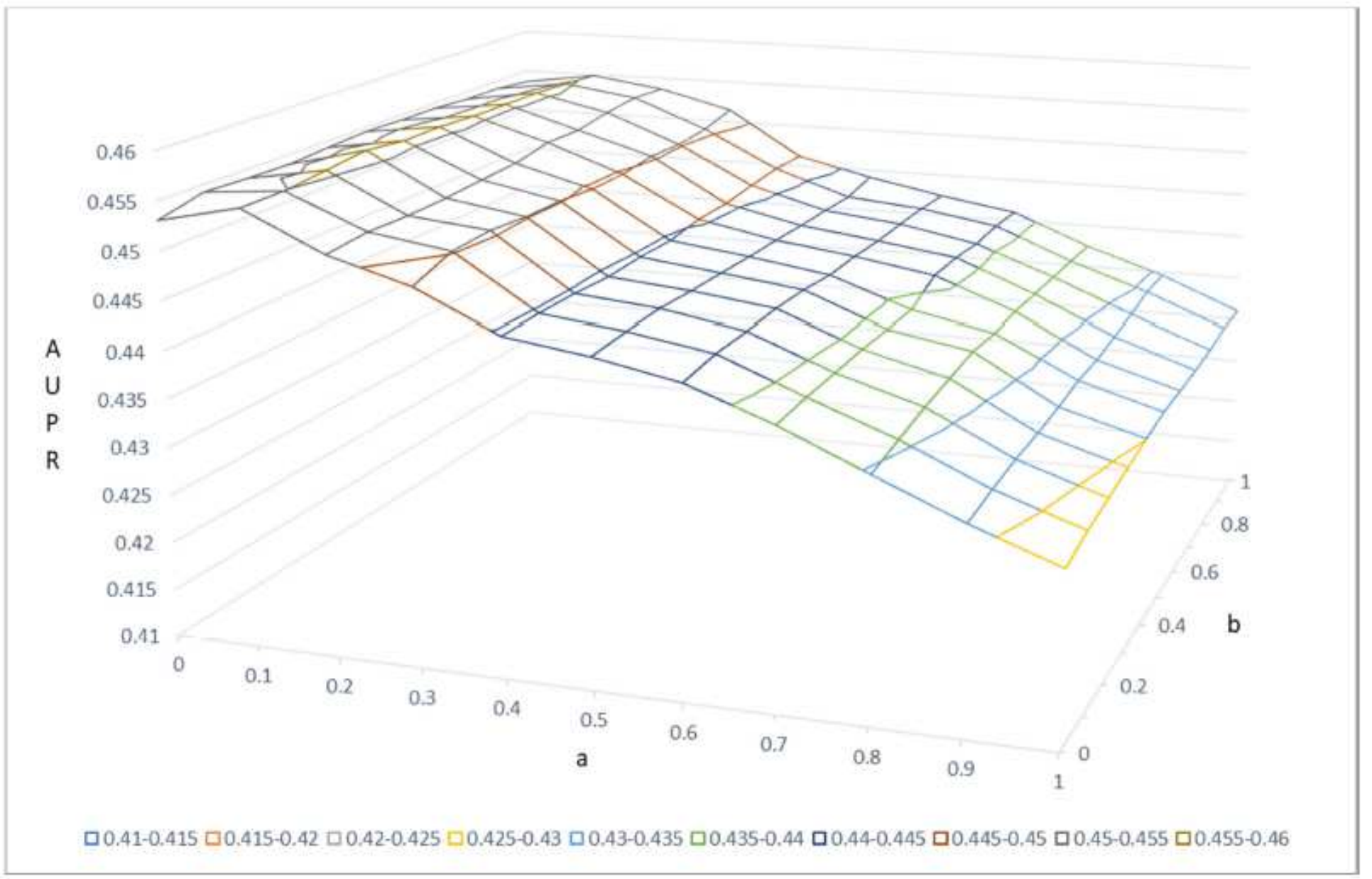

Figure 3

The LOOCV AUPR results of ISKATZWBS with different parameters $₫$ and $\rrbracket$ 


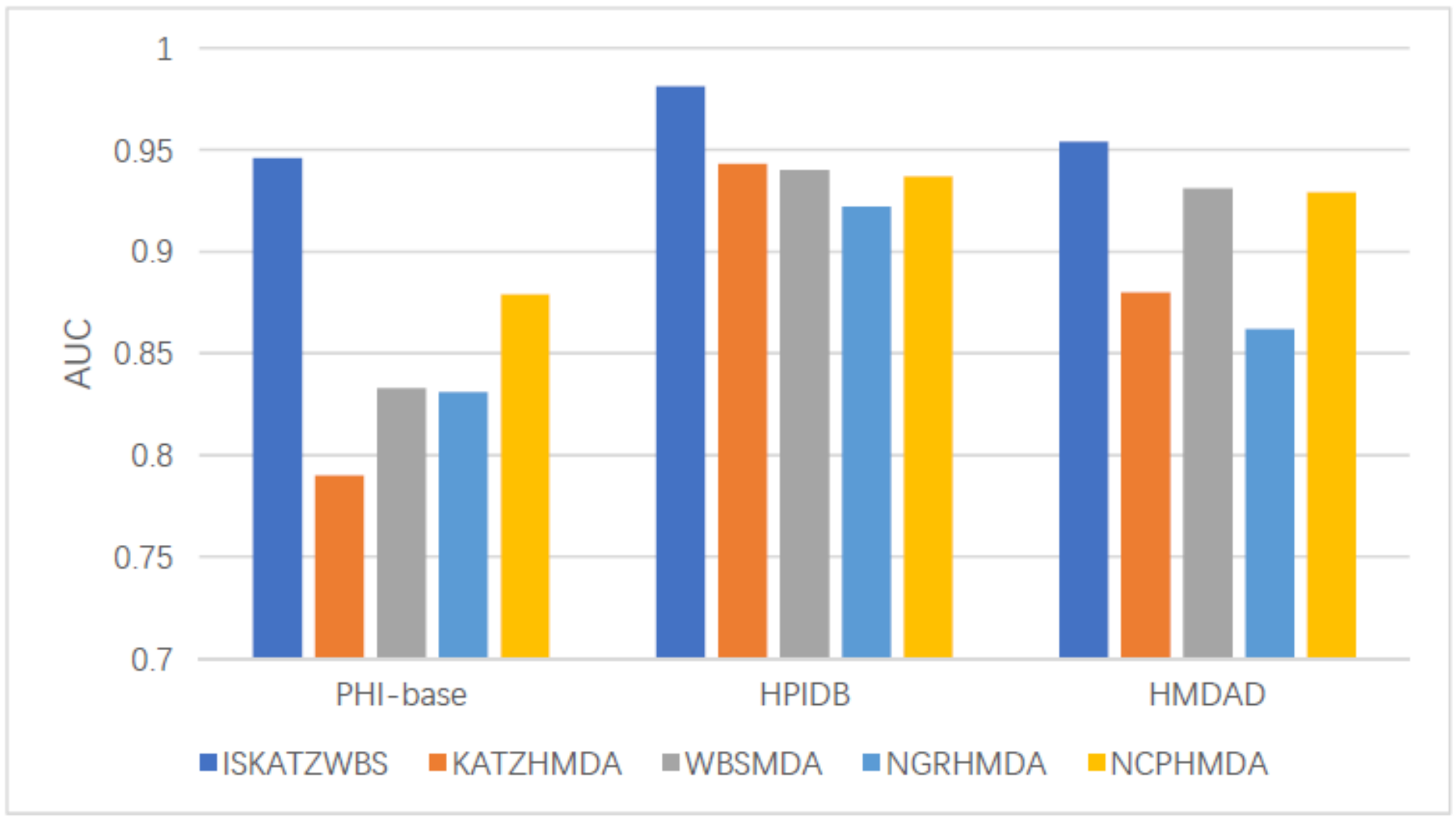

Figure 4

The AUC results of five methods on three data

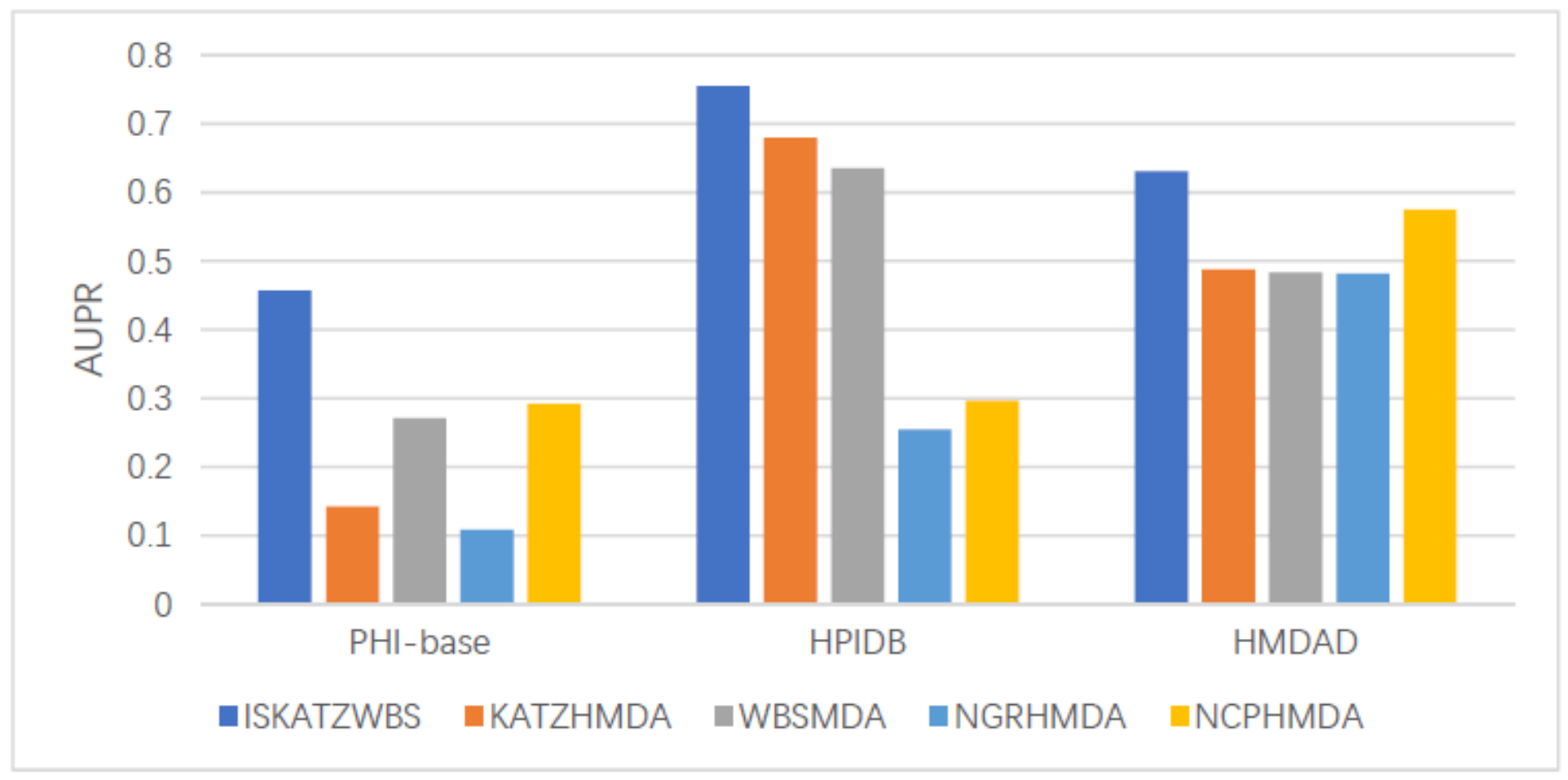

Figure 5

The AUPR results of five methods on three data 